\title{
A Low Complexity Detection/Decoding Algorithm for NB-LDPC Coded PRCPM System
}

\author{
Xiying Liu ${ }^{\mathrm{a}, \mathrm{b}}$, Shancheng Zhao ${ }^{\mathrm{a}}$, Xiao Ma $\mathrm{Ma}^{\mathrm{a}, *}$ \\ ${ }^{a}$ Department of Electronics and Communication Engineering, Sun Yat-sen University, \\ Guangzhou 510275, China \\ ${ }^{b}$ School of Information Engineering, Guangdong University of Technology, Guangzhou \\ 510006, China
}

\begin{abstract}
This paper studies the combination of non-binary low-density paritycheck (NB-LDPC) codes and $M$-ary partial response continuous phase modulation (PRCPM). A low-complexity joint detection/decoding algorithm is proposed, which is referred to as the Max-Log-MAP/X-EMS algorithm. In this joint algorithm, the CPM detector is implemented by the Max-LogMAP algorithm while the LDPC decoder is implemented by the Extended Min-Sum (EMS) algorithms. Three kinds of EMS algorithms, including DEMS, T-EMS, and $M$-EMS algorithms, are compared, which are referred to as $X$-EMS algorithm for convenience. Simulation results show that the Max-Log-MAP/X-EMS algorithm performs as well as the traditional iterative detection/decoding algorithm based on the BCJR algorithm and the QSPA algorithm, but with lower complexity. In addition, comparison of the proposed NB-LDPC coded PRCPM system with the eBCH coded PRCPM
\end{abstract}

\footnotetext{
${ }^{*}$ Corresponding author.

Email addresses: liuxiying@gdut.edu.cn (Xiying Liu), zhaoday@mail2.sysu.edu.cn (Shancheng Zhao), maxiao@mail.sysu.edu.cn (Xiao Ma)
} 
system is given, which shows the performance advantages of our system. Keywords: BCJR algorithm, Iterative decoding, NB-LDPC codes, PRCPM, $X$-EMS algorithms

\section{Introduction}

Continuous phase modulation (CPM) [1] is a bandwidth and energy efficient digital modulation scheme with constant envelope characteristic that makes it particularly appealing for band-limited systems employing nonlinear power amplifiers, such as satellite communication systems. It is well known that the spectral behavior of the partial response CPM (PRCPM) systems is more compact than the full response CPM systems [2, 3]. To achieve high spectral efficiency, high order CPM can be used. This is an appealing property for some communication systems such as satellite video broadcasting and telemetry measures. In [4], it was shown that a CPM modulator can be decomposed into a continuous phase encoder (CPE) followed by a memoryless modulator (MM), where the CPE is a time-invariant convolutional encoder operating on a ring of integers. With the inherent coding property in CPM, serially concatenated CPM (SCCPM) was proposed, and showed a significant improvement in error performance [5, 6]. Several papers considered serial concatenation of CPM with convolutional codes [7], extended $\mathrm{BCH}(\mathrm{eBCH})$ codes [8, 9], binary low density parity-check (LDPC) codes [10], binary LDPC convolutional codes [11] and block Markov superposition transmission of convolutional codes [12]. To the best of our knowledge, most SCCPM systems use binary codes as outer code, while it is more natural to use non-binary codes for M-ary CPM. It is well known that non-binary LDPC 
(NB-LDPC) codes perform better than their binary counterparts when combined with memoryless higher order modulation. But it is not known if this is the case for CPM. This motivates us to investigate the performance of the combination of the NB-LDPC codes and M-ary PRCPM.

NB-LDPC codes were first introduced by Gallager based on modulo arithmetic [13]. In [14], Davey and Mackay presented a class of NB-LDPC codes defined over the finite field $\mathbb{F}_{q}$. They also introduced a $q$-ary sum-product algorithm (QSPA) for decoding NB-LDPC codes. To reduce the decoding complexity, extended min-sum (EMS) algorithms were proposed in [15]. Four kinds of EMS algorithms, which are referred to as $X$-EMS algorithms, are proposed in $[16,17]$. Applications of NB-LDPC codes for combating intersymbol interference and timing recovery can be found in [18, 19].

In this paper, we combine NB-LDPC codes with $M$-ary PRCPM to design a system with power and bandwidth efficiency for data transmission. When NB-LDPC codes are concatenated with M-ary CPM, it becomes necessary to implement the low-complexity detection/decoding algorithms. To reduce the detection/decoding complexity, a Max-Log-MAP/X-EMS algorithm is proposed, in which the detector and the decoder are implemented with the Max-Log-MAP algorithm [20] and X-EMS algorithm [16], respectively. Simulations results show that the Max-Log-MAP/X-EMS algorithm performs as well as the traditional iterative detection/decoding algorithm based on the BCJR algorithm [21] and the QSPA algorithm [14](referred to as BCJR/QSPA), but with reduced complexity.

The remainder of the paper is organized as follows: in Section 2, a detailed description of the system model based on the NB-LDPC codes and $M$-ary 
PRCPM is given. In Section 3, the Max-Log-MAP/X-EMS algorithm is described. In Section 4, simulated results are presented. Some concluding remarks are given in Section 5.

\section{System Description}

\subsection{NB-LDPC Codes}

Let $\mathbb{F}_{q}$ be the finite field with $q$ elements, which is simply denoted by $\mathbb{F}_{q}=\{0,1,2, \cdots, q-1\}$. An NB-LDPC code $\mathcal{C}_{q}[N, K]$ is defined as the null space of a sparse non-binary parity-check matrix $\mathbf{H}=\left[h_{i, j}\right]_{M \times N}$, where $h_{i, j} \in \mathbb{F}_{q}$. A vector $\underline{a}=\left(a_{0}, a_{1}, \cdots, a_{N-1}\right) \in \mathbb{F}_{q}^{N}$ is a codeword if and only if $\mathbf{H} \underline{a}^{\mathrm{T}}=0$.

\subsection{Decomposed Model for $C P M$}

The transmitted signal for CPM system can be described as $[2,3]$

$$
z(t, \mathbf{v})=\sqrt{\frac{2 E_{s}}{T}} \cos \left(2 \pi f_{0} t+\psi(t, \mathbf{v})+\psi_{0}\right), j T<t<(j+1) T
$$

where $\psi(t, \mathbf{v})=2 \pi h \sum_{i=0}^{\infty} v_{i} q(t-i T)$ is the information-carrying phase. Here, $E, T, f_{0}$ and $\psi_{0}$ denote symbol energy, symbol time, carrier frequency and an initial phase shift, respectively. Parameter $h=K / P$ is a rational number and it is referred to as the modulation index. The sequence $\mathbf{v}$ is an $M$ ary symbol sequence with $v_{i} \in\{ \pm 1, \pm 3, \cdots, \pm(M-1)\}$. The $q(t)$ is phase pulse, which is usually defined as the integral of a frequency pulse $g(t)$. The frequency pulse has the length of $L$ symbol intervals. If $L=1$, the scheme is called full response CPM. In other cases $(L>1)$, the scheme is called partial 
response CPM. A CPM scheme is then defined by specifying its parameters $M, h, L$, and the frequency pulse $g(t)$.

In [4] it was shown that the CPM modulator can be described by a concatenation of a time-invariant $\mathrm{CPE}$ with a time-invariant $\mathrm{MM}$ if $\psi(t, \mathbf{v})$ in (1) is replaced by the so-called titled phase $\bar{\psi}(t, \overline{\mathbf{v}})$ with

$$
\begin{aligned}
\bar{\psi}(\tau+j T, \overline{\mathbf{v}})= & {\left[2 \pi h\left[\sum_{i=0}^{j-L} \bar{v}_{i}\right] \bmod P\right.} \\
& \left.+4 \pi h \sum_{i=0}^{L-1} \bar{v}_{j-i} q(\tau+i T)+W(\tau)\right] \bmod 2 \pi, 0 \leq \tau \leq T
\end{aligned}
$$

where $\bar{v}_{i}=\left[v_{i}+(M-1)\right] / 2, W(\tau)$ represents the data-independent terms, and mod denotes the modulo operator. Hence, the transmitted signal in the $j$ th interval is determined by the current data symbol $\bar{v}_{j}$, the previous data symbols $\bar{v}_{j-1}, \cdots, \bar{v}_{j-L+1}$ and the accumulated phase state $\theta_{j}=$ $\left[\sum_{i=0}^{j-L} \bar{v}_{i}\right] \bmod P$. Therefore, the number of different signals in CPM is $P M^{L}$.

Since the CPE is a convolutional code, it can be represented by a timeinvariant trellis. The trellis has $N$ stages, which are indexed by $0 \leq j \leq N-1$. The $j$ th stage corresponds to the $j$ th coded symbol $v_{j}$. At each stage, there are $P M^{L-1}$ states, which are simply indexed by $0 \leq s \leq P M^{L-1}-1$. The collection of the states at the $j$ th stage is denoted by $\mathcal{S}_{j}$. Emitting from each state, there are $M$ branches. Each branch in the $j$ th stage is specified by a 4 tuple $\underline{b} \triangleq\left(s_{j}, v_{j}, x_{j}, s_{j+1}\right)$, where $v_{j} \in \mathbb{F}_{q}$ is the $j$ th possible coded symbol that takes the state from $s_{j}$ into $s_{j+1}$ and results in the output $x_{j}$. The collection of branches in the $j$ th stage is denoted by $\mathcal{B}_{j}$. We have $\left|\mathcal{B}_{j}\right|=P M^{L}$. 

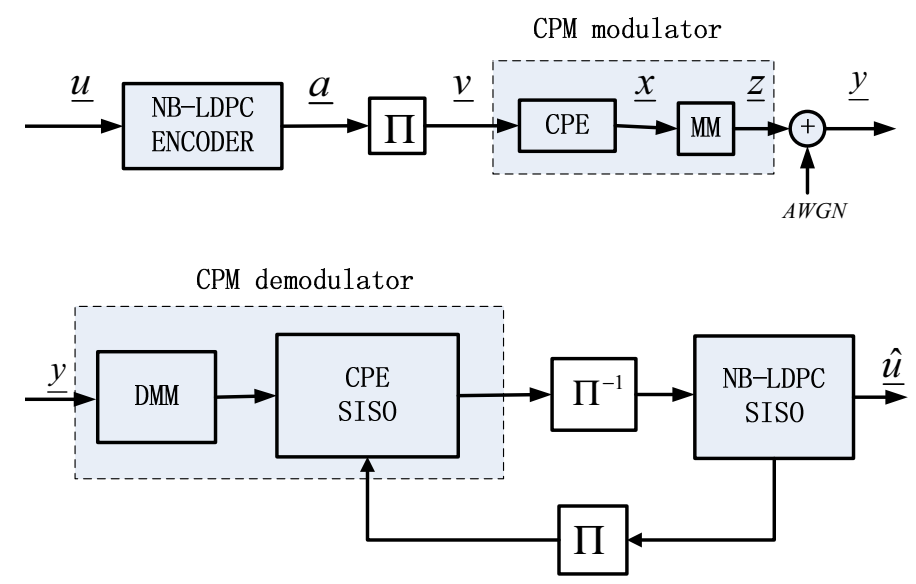

Figure 1: The system model of NB-LDPC coded $M$-ary PRCPM.

\subsection{System Model}

The system model of NB-LDPC coded $M$-ary PRCPM is shown in Fig. 1. We always assume that $q=M$.

Encoding: The information sequence $\underline{u}=\left(u_{0}, u_{1}, \cdots, u_{K-1}\right) \in \mathbb{F}_{q}^{K}$ is encoded by $q$-ary LDPC encoder into a codeword $\underline{a}=\left(a_{0}, a_{1}, \cdots, a_{N-1}\right) \in$ $\mathbb{F}_{q}^{N}$.

Symbol Interleaving: Interleave $\underline{a}=\left(a_{0}, a_{1}, \cdots, a_{N-1}\right)$ into $\underline{v}=\left(v_{0}, v_{1}, \cdots, v_{N-1}\right)$ based on the inteleaver $\Pi$. The inverse of $\Pi$ is denoted as $\Pi^{-1}$.

$C P E$ : Encode $\underline{v}=\left(v_{0}, v_{1}, \cdots, v_{N-1}\right)$ into $\underline{x}=\left(x_{0}, x_{1}, \cdots, x_{N-1}\right)$ by the encoding algorithm of the CPE.

$M M$ : The $\underline{x}=\left(x_{0}, x_{1}, \cdots, x_{N-1}\right)$ is mapped into the transmitted waveform sequence $\underline{z}=\left(z_{0}, z_{1}, \cdots, z_{N-1}\right)$ according to the mapping rule of MM, where $z_{j}, 0 \leq j \leq N-1$, is only determined by $x_{j}$.

Channel: Assume that the waveform sequence $\underline{z}$ is transmitted through an additive white Gaussian noise (AWGN) channel, where the noise samples are independently distributed (i.i.d) Gaussian random variables with 
distribution $\mathcal{C N}\left(0,2 \sigma^{2}\right)$. The received waveform sequence can be denoted by $\underline{y}=\left(y_{0}, y_{1}, \cdots, y_{N-1}\right)$ with $y_{j}=z_{j}+w_{j}, 0 \leq j \leq N-1$, where $y_{j}$ and $w_{j}$ are the received waveform and the noise waveform in the $j$ th interval, respectively.

Detection/Decoding: Upon receiving $\underline{y}$, the receiver attempts to recover the transmitted data $\underline{u}$. Firstly, the memoryless demodulator (referred to as DMM) computes the possibility information about $\underline{x}$ for decoding. Secondly, the iterative receiver performs decoding iterations between the inner softin soft-out(SISO) decoder, which operates on the CPE trellis, and the outer SISO decoder, which operates on the outer NB-LDPC code trellis. The iterative detection/decoding algorithm described in the next section is performed by following the turbo principal [22].

\section{The Detection/Decoding algorithm}

The detection/decoding algorithm for the NB-LDPC coded PRCPM system can be described as an iterative message processing/passing algorithm over the normal graph shown in Fig. 2. The normal graph has six types of nodes (constraints): $N$ nodes of MM (M-node), $N$ trellis nodes of CPE (Tnode), a node of interleaver ( $\Pi$-node), $N$ variable nodes (V-node), $M$ check nodes (C-node), and $\delta$ H-nodes, where $\delta$ denotes the number of nonzero elements in the parity-check matrix $\mathbf{H}$. In the normal graph: (1) the M-node represents the constraint introduced by the mapping rule of MM; (2) the T-node represents the constraint that $x_{j}$ must be a codeword of the CPE that corresponds to $v_{j}, 0 \leq j \leq N-1$; (3) the $\Pi$-node represents the interleaver, which interleaves or de-interleaves the input messages; (4) a V-node 


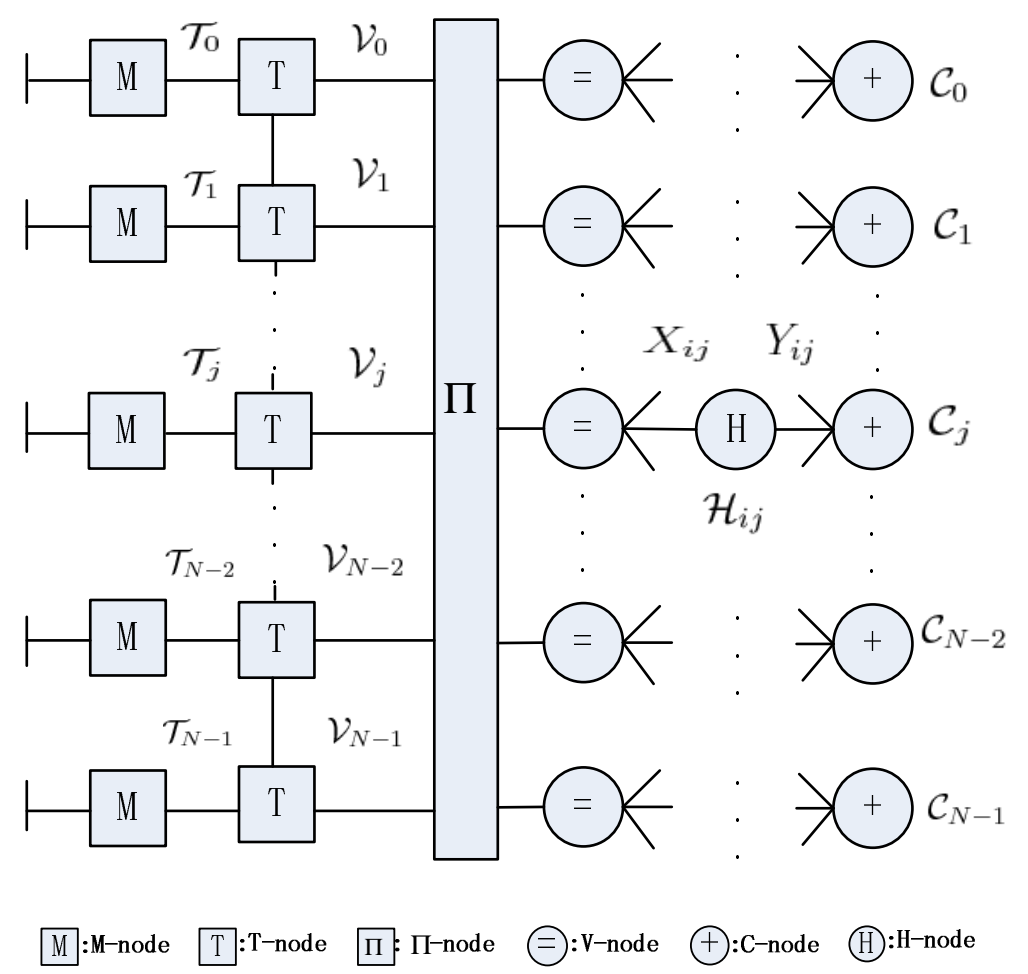

Figure 2: Normal graph of a NB-LDPC codes PRCPM system.

corresponds to a column of $\mathbf{H}$; (5) a C-node corresponds to a row of $\mathbf{H}$; and (6) the H-node corresponds to $h_{i, j} \neq 0$.

Let $\mathrm{W}$ be a discrete random variable taking on values over $\mathcal{W}$. We use $P_{\mathrm{W}}(w), w \in \mathcal{W}$ to denote its probability mass function (pmf). Let $\mathrm{W}$ denote the variable on the edge connecting the node $\mathcal{A}_{1}$ and $\mathcal{A}_{2}$ in the normal graph. We will use $L_{\mathrm{W}}^{\left(\mathcal{A}_{1} \rightarrow \mathcal{A}_{2}\right)}$ to denote the log-domain message from $\mathcal{A}_{1}$ to $\mathcal{A}_{2}$. A log-domain message is defined as the logarithm of the probability vector or its linear transform. In this paper, the log-domain messages are calculated by $L_{\mathrm{W}}(w)=\left[a_{0} \log P_{\mathrm{W}}(w)+a_{1}\right], w \in \mathcal{W}$, where $a_{0}>0, a_{1} \in \mathbb{R}$ are two constants and $[x]$ represents the integer that is closest to $x \in \mathbb{R}$. We can confine the 
range of $L_{\mathrm{W}}(w)$ to be $\left[0,2^{p}-1\right]$ by properly choosing $a_{0}$ and $a_{1}$. In this case $L_{\mathrm{W}}(w)$ is also referred to as a $p$-bit possibility function.

\subsection{Review of the X-EMS Algorithms}

The QSPA has computational complexity $O\left(q^{2}\right)$ and its straightforward implementation has a very high computational complexity. To reduce the computational complexity, Declercq et al. proposed the EMS algorithm [15] by considering only dominant terms. In [16], the $X$-EMS (the $M$-EMS algorithm, the $T$-EMS algorithm and the $D$-EMS algorithm) were proposed and described in a unified way as reduced-search trellis algorithms. The $X$-EMS algorithms use log-domain messages and message truncation techniques. Using the message-truncation techniques [16], the $M$-EMS algorithm was described as a reduced-search trellis algorithm that retains only those states/branches at each trellis section with the $M$ largest metrics; the $D$-EMS algorithm retains only those states/branches at each trellis section whose metrics deviate from the maximum one within a designated parameter $D$; while the $T$-EMS algorithm retains only those states/branches at each trellis section whose metrics exceed a designated threshold $T$.

Given the log-domain message $L_{Y_{i j}}^{\left(\mathcal{H}_{i j} \rightarrow \mathcal{C}_{i}\right)}(y), y \in \mathbb{F}_{q}$, we can partition the finite field $\mathbb{F}_{q}$ into $\mathcal{F}$ and $\mathbb{F}_{q}-\mathcal{F}$. Three message truncation techniques in [16] are as follows:

$$
\begin{gathered}
\mathcal{F}_{M}=\left\{y \in \mathbb{F}_{q} \mid L_{Y_{i j}}^{\left(\mathcal{H}_{i j} \rightarrow \mathcal{C}_{i}\right)}(y) \text { is one of the } M \text { largest components of } L_{Y_{i j}}^{\left(\mathcal{H}_{i j} \rightarrow \mathcal{C}_{i}\right)}\right\}, \\
\mathcal{F}_{T}=\left\{y \in \mathbb{F}_{q} \mid L_{Y_{i j}}^{\left(\mathcal{H}_{i j} \rightarrow \mathcal{C}_{i}\right)}(y) \geq T\right\}
\end{gathered}
$$


and

$$
\mathcal{F}_{D}=\left\{y \in \mathbb{F}_{q} \mid L_{\text {max }}-L_{Y_{i j}}^{\left(\mathcal{H}_{i j} \rightarrow \mathcal{C}_{i}\right)}(y) \leq D\right\}
$$

where $L_{\text {max }}$ denotes the largest component of $L_{Y_{i j}}^{\left(\mathcal{H}_{i j} \rightarrow \mathcal{C}_{i}\right)}$ and $D$ is a designated parameter.

\subsection{Demodulation at $M$-node}

Let $\mathcal{X}$ be the signal collection corresponding to the MM input $x_{j}$. The cardinality of $\mathcal{X}$ is $|\mathcal{X}|=P M^{L}$. Denote the mapping rule of $\mathrm{MM}$ as $\Psi$, then we have $\Psi\left(x_{j}\right)=z_{j}$ in the $j$ th interval. In this paper, we use the signal space [1] based simulation method. Each waveform at a transmission interval is represented as a linear combination of a set of othonormal bases, which are obtained by Gram-Schmidt procedure [23]. Assume that the number of orthonormal bases sufficiently spanning the signal space is $\rho$. Then the transmitted waveform $z_{j}$ and received waveform $y_{j}$ in the $j$ th interval can be represented by $\rho$ dimensional complex vectors in simulation. Upon receiving $y_{j}$, the demodulation at $M$-node aims to compute the possibility $\mathrm{P}_{X_{j} \mid Y_{j}}\left\{x \mid y_{j}\right\}, x \in \mathcal{X}$ for detection.

In the BCJR/QSPA algorithm, the possibility is computed by

$$
\mathrm{P}_{X_{j} \mid Y_{j}}\left\{x \mid y_{j}\right\}=\alpha_{j} \exp \left\{-\frac{\left\|y_{j}-\Psi(x)\right\|^{2}}{2 \sigma^{2}}\right\}, x \in \mathcal{X}
$$

where $\|\cdot\|^{2}$ representes the squared Euclidean norm, $\alpha_{j}$ is the normalized factor to ensure $\sum_{x \in \mathcal{X}} \mathrm{P}_{X_{j} \mid Y_{j}}\left\{x \mid y_{j}\right\}=1$.

In the max-log-MAP / $X$-EMS algorithm, we compute the possibility function corresponding to $\mathrm{P}_{X_{j} \mid Y_{j}}\left\{x \mid y_{j}\right\}, x \in \mathcal{X}$. The algorithm is described as follows. 
Algorithm 1. Possibility function calculation

Given the received waveform $y_{j}$, the range $2^{p}-1$ and the maximum allowable squared Euclidean distance $d_{\max }$ for quantization. For $j=0,1, \cdots, N-$ 1:

Step 1: Calculate $d(x)=\left\|y_{j}-\Psi(x)\right\|^{2}$ for each $x \in \mathcal{X}$.

Step 2: If $d(x)>d_{\max }$, set $d(x)=d_{\max }$.

Step 3: For each $x \in \mathcal{X}$, calculate

$$
L_{X_{j}}^{\mid \rightarrow \mathcal{T}_{j}}(x)=\left[\frac{d_{\max }-d(x)}{d_{\max }} \times\left(2^{p}-1\right)\right], x \in \mathcal{X}
$$

It is easy to verify that $L_{X_{j}}^{\mid \rightarrow \mathcal{T}_{j}}(x)$ is a $p$-bit possibility function. For the least possible element $x$, we have $d(x)=d_{\max }$ and $L_{X_{j}}^{\mid \rightarrow \mathcal{T}_{j}}(x)=0$. While for the most possible element $x, d(x)$ will tend to zero and $L_{X_{j}}^{\mid \rightarrow \mathcal{T}_{j}}(x)$ will tend to $2^{p}-1$. As a result, the detector requires only integer operations.

\subsection{The iterative detection/decoding algorithm}

In this paper, a reduced complexity Max-Log-MAP/X-EMS algorithm is proposed. The traditional BCJR/QSPA algorithm is taken as benchmark for comparison.

Algorithm 2. The Max-Log-MAP/X-EMS algorithm

- Memoryless demodulation and initialization: Assuming that $\underline{y}$ have been received, compute $L_{X_{k}}^{\mid \rightarrow \mathcal{T}_{k}}\left(x_{k}\right)$ for $0 \leq k \leq N-1$ using Algorithm 1. Given a truncation rule $\mathcal{F}$, set a maximum iteration number $I_{\text {max }}$. For all $\mathcal{V}_{j}, 0 \leq j \leq N-1$, and $x \in \mathbb{F}_{q}$, set $L_{V_{j}}^{\left(\mathcal{V}_{j} \rightarrow \mathcal{T}_{j}\right)}(x)=0$, $L_{X_{i j}}^{\left(\mathcal{H}_{i j} \rightarrow \mathcal{V}_{j}\right)}(x)=0$. 
- Iteration: For $I=1,2, \cdots, I_{\max }$

1) T-node: Firstly assign the integer metric $L_{k}\left(b_{k}\right)=L_{X_{k}}^{\left(\mid \rightarrow \mathcal{T}_{k}\right)}\left(x_{k}\right)+$ $L_{V_{k}}^{\left(\mathcal{V}_{k} \rightarrow \mathcal{T}_{k}\right)}\left(v_{k}\right)$ for each branch $b_{k}=\left(s_{k}, v_{k}, x_{k}, s_{k+1}\right), 0 \leq k<N$. Then perform the Max-Log-MAP algorithm to obtain $L_{V_{k}}^{\left(\mathcal{T}_{k} \rightarrow \mathcal{V}_{k}\right)}$.

2) П-node: De-interleave $L_{V_{k}}^{\left(\mathcal{T}_{k} \rightarrow \mathcal{V}_{k}\right)}$ into $L_{V_{j}}^{\left(\mathcal{T}_{j} \rightarrow \mathcal{V}_{j}\right)}$, where $k$ is the interleaved value of $j$, i.e, $j=\Pi^{-1}(k)$.

3)V-node: For all V-nodes, calculate the extrinsic message from $\mathcal{V}_{j}$ to its adjacent H-nodes by $L_{X_{i j}}^{\left(\mathcal{V}_{j} \rightarrow \mathcal{H}_{i j}\right)}(x)=L_{V_{j}}^{\left(\mathcal{T}_{j} \rightarrow \mathcal{V}_{j}\right)}(x)+\sum_{k \neq i} L_{X_{k j}}^{\left(\mathcal{H}_{k j} \rightarrow \mathcal{V}_{j}\right)}(x), x \in$ $\mathbb{F}_{q}$.

4) H-node: For all H-nodes, permute the messages $L_{Y_{i j}}^{\left(\mathcal{H}_{i j} \rightarrow \mathcal{C}_{i}\right)}$ by $L_{Y_{i j}}^{\left(\mathcal{H}_{i j} \rightarrow \mathcal{C}_{i}\right)}(y)=$ $L_{X_{i j}}^{\left(\mathcal{V}_{j} \rightarrow \mathcal{H}_{i j}\right)}\left(h_{i j}^{-1} y\right), y \in \mathbb{F}_{q}$.

5) C-node: For all C-nodes, calculate the extrinsic messages $L_{Y_{i j}}^{\left(\mathcal{C}_{i} \rightarrow \mathcal{H}_{i j}\right)}(y)$ according to the truncation rule $\mathcal{F}$.

6) H-node: For all H-nodes, permute the messages $L_{X_{i j}}^{\left(\mathcal{H}_{i j} \rightarrow \mathcal{V}_{j}\right)}$ by $L_{X_{i j}}^{\left(\mathcal{H}_{i j} \rightarrow \mathcal{V}_{j}\right)}(x)=$ $L_{Y_{i j}}^{\left(\mathcal{C}_{i} \rightarrow \mathcal{H}_{i j}\right)}\left(h_{i j} x\right), x \in \mathbb{F}_{q}$.

7) V-node: For the $\mathrm{V}$-node $\mathcal{V}_{j}(0 \leq j \leq N-1)$, calculate $L_{V_{j}}(x)=$ $L_{V_{j}}^{\left(\mathcal{T}_{j} \rightarrow \mathcal{V}_{j}\right)}(x)+\sum_{i \in \mathcal{M}_{j}} L_{X_{i j}}^{\left(\mathcal{H}_{i j} \rightarrow \mathcal{V}_{j}\right)}(x)$ and find $\hat{v}_{j}$ by $\hat{v}_{j}=\arg \max _{x \in \mathbb{F}_{q}} L_{V_{j}}(x)$. If $H \underline{\hat{v}}^{T}=\underline{0}$, output $\underline{\hat{v}}$ as the estimated codeword and exit the iteration. If $H \underline{\hat{v}}^{T} \neq \underline{0}$, calculate $L_{V_{j}}^{\left(\mathcal{V}_{j} \rightarrow \mathcal{T}_{j}\right)}(x)=L_{V_{j}}(x)-L_{V_{j}}^{\left(\mathcal{T}_{j} \rightarrow \mathcal{V}_{j}\right)}(x)$ for next iteration.

8) П-node: Interleave $L_{V_{j}}^{\left(\mathcal{T}_{j} \rightarrow \mathcal{V}_{j}\right)}$ into $L_{V_{k}}^{\left(\mathcal{T}_{k} \rightarrow \mathcal{V}_{k}\right)}$ with $k=\Pi(j)$.

Remark: Note that the proposed algorithm requires only integer operations and finite field operations. 


\section{Simulation Results}

The spectral efficiency (SE) of coded CPM can be approximated by [6]

$$
\mathrm{SE} \approx R \cdot \frac{\log _{2} M}{B_{u} \cdot T} \mathrm{bit} / \mathrm{s} / \mathrm{Hz}
$$

where $R$ is the rate of the outer code and $B_{u}$ is the normalized double-sided uncoded bandwidth. Note that $B_{u}$ depends on $M, L$, the pulse type, and the modulation index $h$.

In Example 1, we compare the frame error rates (FER) of different SCCPM systems with similar spectral efficiency. In Example 2 and Example 3, we compare the complexity of Max-Log-MAP/X-EMS algorithm with that of BCJR/QSPA algorithm. The performance is measured by the FER v.s $E_{b} / N_{0}$ with

$$
\frac{E_{b}}{N_{0}}=10 \log _{10}\left[\frac{E_{s}}{N_{0} R \log _{2} M}\right] .
$$

The complexity is measured by the ratio of its computational complexity to that of the BCJR/QSPA.

Apparently, the Max-Log-MAP/X-EMS algorithm requires less operation than BCJR/QSPA at each iteration. However, they may require more iterations to converge. For a fair comparison, we take

$$
\frac{\text { Total number of operations of a given algorithm }}{\text { Total number of operations of the BCJR/QSPA algorithm }}
$$

as the complexity measurement. Note that the statistical mean (averaging over frames) of the total number of operations involved in all iterations for decoding one frame is used in (7). Also note that the ratio in (7) only give a 
rough comparison, as different algorithms require different operations. It is worth mentioning that, for the detection of CPM, the Max-Log-MAP algorithm requires the number of operation as much as the BCJR algorithm. However, in practice, the Max-Log-MAP algorithm is more often implemented for its low complexity.

In the following Examples, we consider raised cosine (RC) family of frequency pulses $g(t)$ with $\mathrm{M}=\{4,8,16\}$ and $L=\{2,3\}$ for CPM, denoted as $\mathrm{M} L \mathrm{RC}$. For instance Q3RC sands for quaternary $L=3$ raised cosine, O2RC sands for octal $L=2$ raised cosine and H2RC sands for hexadecimal $L=2$ raised cosine. The maximum number of global and local iteration is 80 and 1 , respectively.

Example 1. 4-ary $L D P C$ coded Q3RC system

The performances of 4-ary LDPC coded Q3RC system are evaluated in this example. For Q3RC, the modulation index $h$ is $1 / 7$. We select two 4-ary LDPC codes with similar rates for simulation. One is a column-scaled (CS) NB-LDPC code [24] $\mathcal{C}_{4}[645,518]$, the other is a quasi-cyclic (QC) NB-LDPC code $\mathcal{C}_{4}[645,516]$. Their parity-check matrices have row weight 15 and column weight 3. The SE of this NB-LDPC coded Q3RC is about $2.25 \mathrm{bit} / \mathrm{s} / \mathrm{Hz}$. A comparable concatenated system based on eBCH code was proposed in [9], where the authors showed the advantages of the concatenated system based on $[64,51] \mathrm{eBCH}$ code and Q3RC in terms of convergence rate and error floor. The FER performances of 4-ary LDPC coded Q3RC systems and the binary eBCH coded Q3RC system are shown in Fig. 3. It can be seen that an extra coding gain of about $1.0 \mathrm{~dB}$ at $\mathrm{FER}=10^{-5}$ is obtained with 4-ary LDPC codes. 


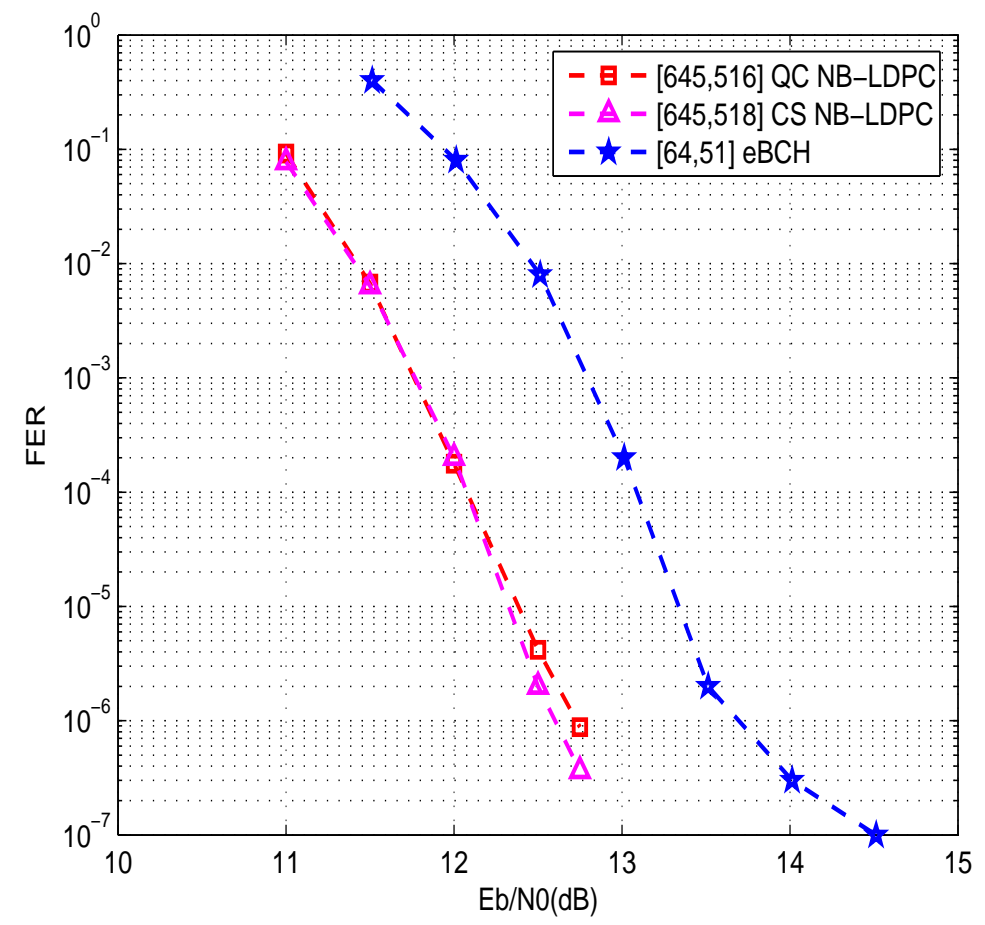

Figure 3: FER performance of serially concatenated Q3RC, $h=1 / 7$ for spectral efficiency $2.25 \mathrm{bit} / \mathrm{s} / \mathrm{Hz}$. 
Remark: In Fig. 3, the curve for $[64,51]$ eBCH is obtained by shifting the curve in Fig. 5 of [9] to right by $1.51332 \mathrm{~dB}$. This is due to the difference between our definition of $E_{b} / N_{0}$ with that in [9]. In [9], the definition of $E_{b} / N_{0}$ is

$$
\frac{E_{b}}{N_{0}}=10 \log _{10}\left[\frac{E_{s}}{N_{0} R \log _{2} M B_{u} T}\right] .
$$

Example 2. 8-ary $L D P C$ coded O2RC system

The performances of 8-ary LDPC coded O2RC system are evaluated in this example. For O2RC, the modulation index $h$ is $1 / 2$. The simulated code is the 8-ary LDPC code $\mathcal{C}_{8}[225,173]$ of rate 0.768 , which is a CS LDPC code constructed by finite fields $[24,25]$. The corresponding parity-check matrix has row weight 14 and column weights 3 and 4 . The parameters of the Max-Log-MAP / $X$-EMS algorithms are as follows.

1) For the $D$-EMS algorithm, $D_{s}=100, D_{b}=100$. For the $T$-EMS algorithm, $T_{s}=50, T_{b}=40$. For the $M$-EMS algorithm, $M=4$.

2) Like in [16], we use the scaling factor $\xi$ to correct the overestimation. The $\xi$ of the $D$-EMS algorithm, $T$-EMS algorithm, $M$-EMS algorithm are 0.6, 0.6 and 0.7 , respectively.

3) The squared Euclidean distances are quantized with $p=9, d_{\max }=10$.

The error performances and the complexity ratios of different algorithms for the $\mathcal{C}_{8}[225,173]$ coded O2RC system are shown in Fig. 4 and Fig. 5, respectively. From Fig. 4, it can be seen that the Max-Log-MAP/X-EMS algorithms have almost the same performance and suffer from performance degradation about $0.1 \mathrm{~dB}$ compared with BCJR/QSPA algorithm. From Fig. 5, it 


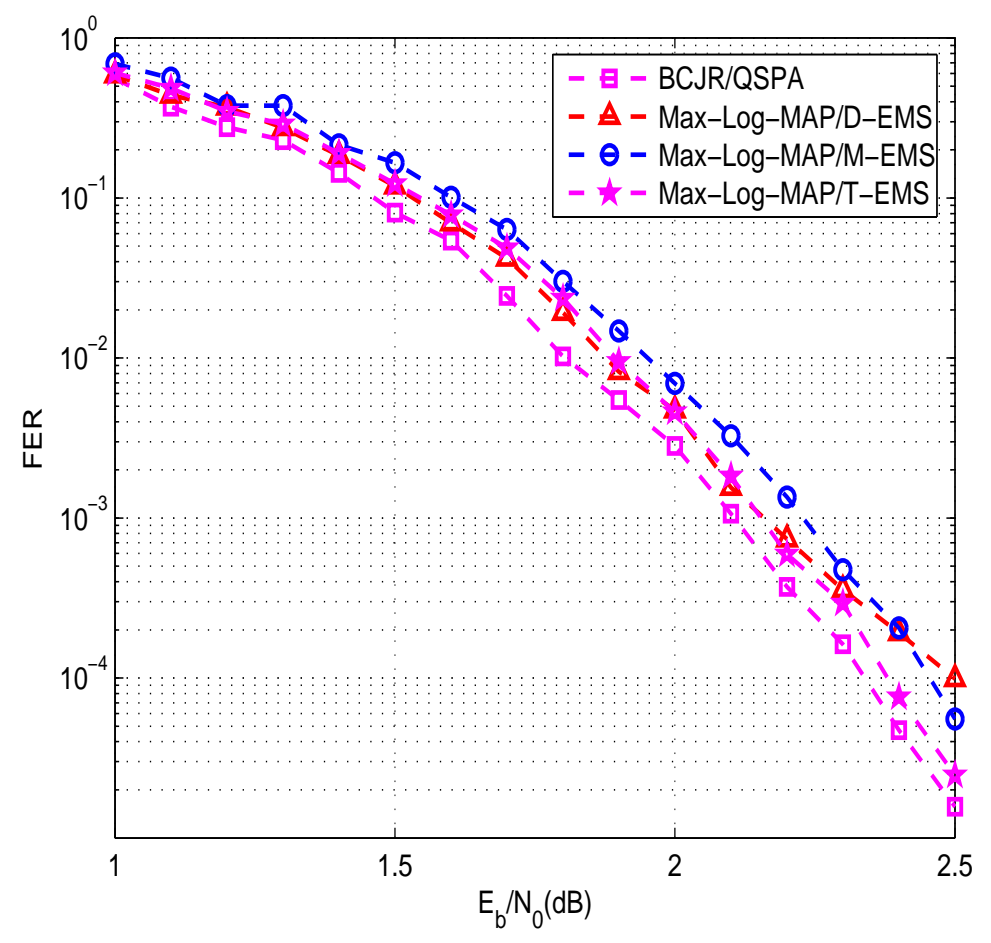

Figure 4: FER performance of different detection/decoding algorithms for the $\mathcal{C}_{8}[225,173]$ coded O2RC system. 


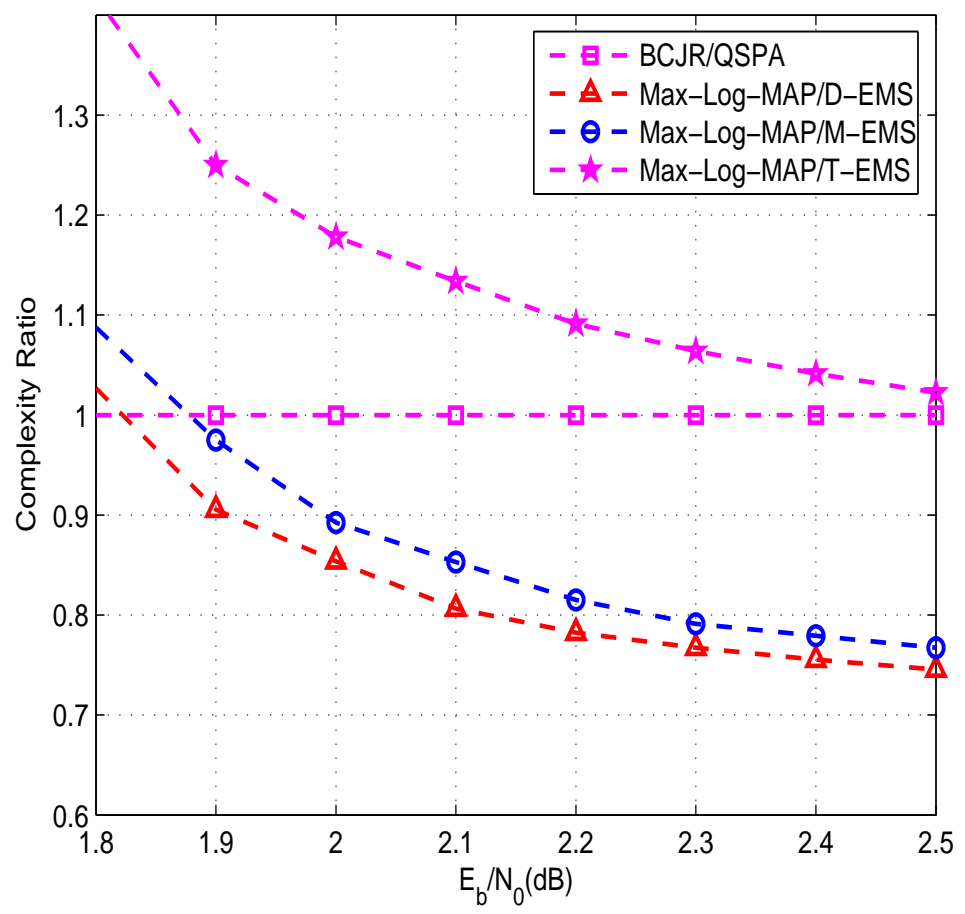

Figure 5: Complexity ratios of different detection/decoding algorithms for the $\mathcal{C}_{8}[225,173]$ coded O2RC system. 
can be seen that the Max-Log-MAP/D-EMS is the simplest one with complexity ratio about 0.75 at $E_{b} / N_{0}=2.5 \mathrm{~dB}$. We also notice that the MaxLog-MAP/T-EMS algorithm has a higher complexity than BCJR/QSPA in the lower $E_{b} / N_{0}$ region. This is because the complexity reduction per iteration is not enough to compensate the complexity increase caused by the extra iterations. However, the complexity ratio becomes lower with increas$\operatorname{ing} E_{b} / N_{0}$.

Example 3. 16-ary LDPC coded H2RC system

The performances of 16-ary LDPC coded H2RC system are evaluated in this example. For H2RC, the modulation index $h$ is $1 / 2$. All the parameters for the code are the same as in Example 2 except for the $M=16$. The parameters of the Max-Log-MAP/X-EMS algorithms are as follows.

1) For the $D$-EMS algorithm, $D_{s}=100, D_{b}=100$. For the $T$-EMS algorithm, $T_{s}=50, T_{b}=50$. For the $M$-EMS algorithm, $M=8$.

2) The $\xi$ of the $D$-EMS algorithm, $T$-EMS algorithm, $M$-EMS algorithm are $0.6,0.6$ and 0.7 , respectively.

3) The squared Euclidean distances are quantized with $p=9, d_{\max }=10$.

The error performances and the complexity ratios of different algorithms for the $\mathcal{C}_{16}[225,173]$ coded H2RC system are shown in Fig. 6 and Fig. 7, respectively. We find that the error performance and complexity ratios have a similar behavior to that in Example 2. From Fig. 7, it can be seen that the Max-Log-MAP/D-EMS is the simplest one with complexity ratio about 0.5 at $E_{b} / N_{0}=1.8 \mathrm{~dB}$. In Fig. 8, we present the average iteration numbers 


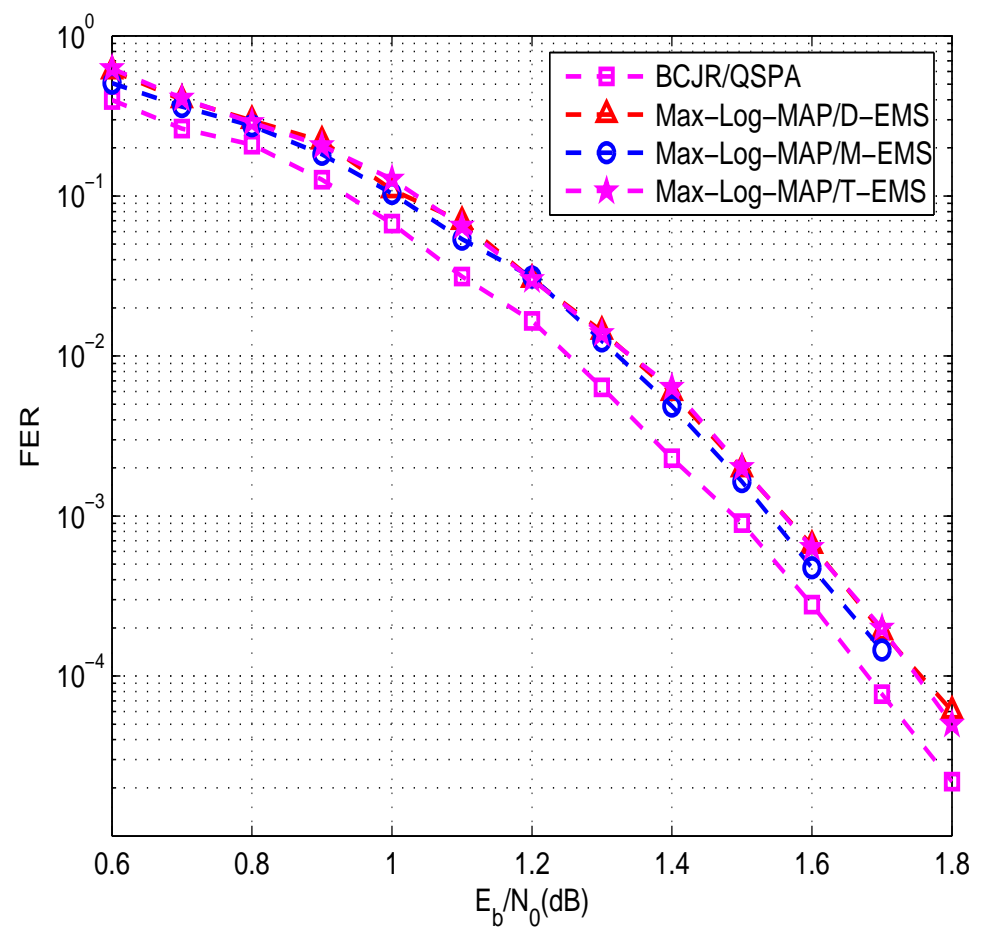

Figure 6: FER performance of different detection/decoding algorithms for the $\mathcal{C}_{16}[225,173]$ coded H2RC system. 


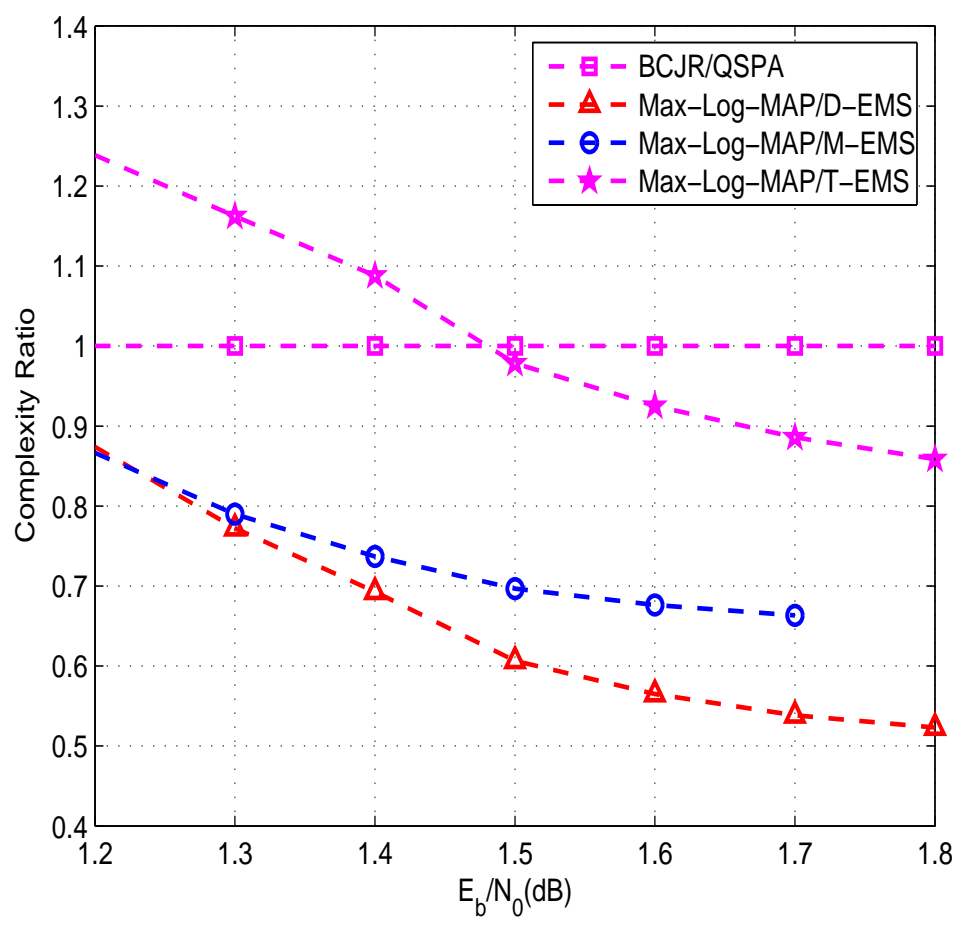

Figure 7: Complexity ratios of different detection/decoding algorithms for the $\mathcal{C}_{16}[225,173]$ coded H2RC system. 


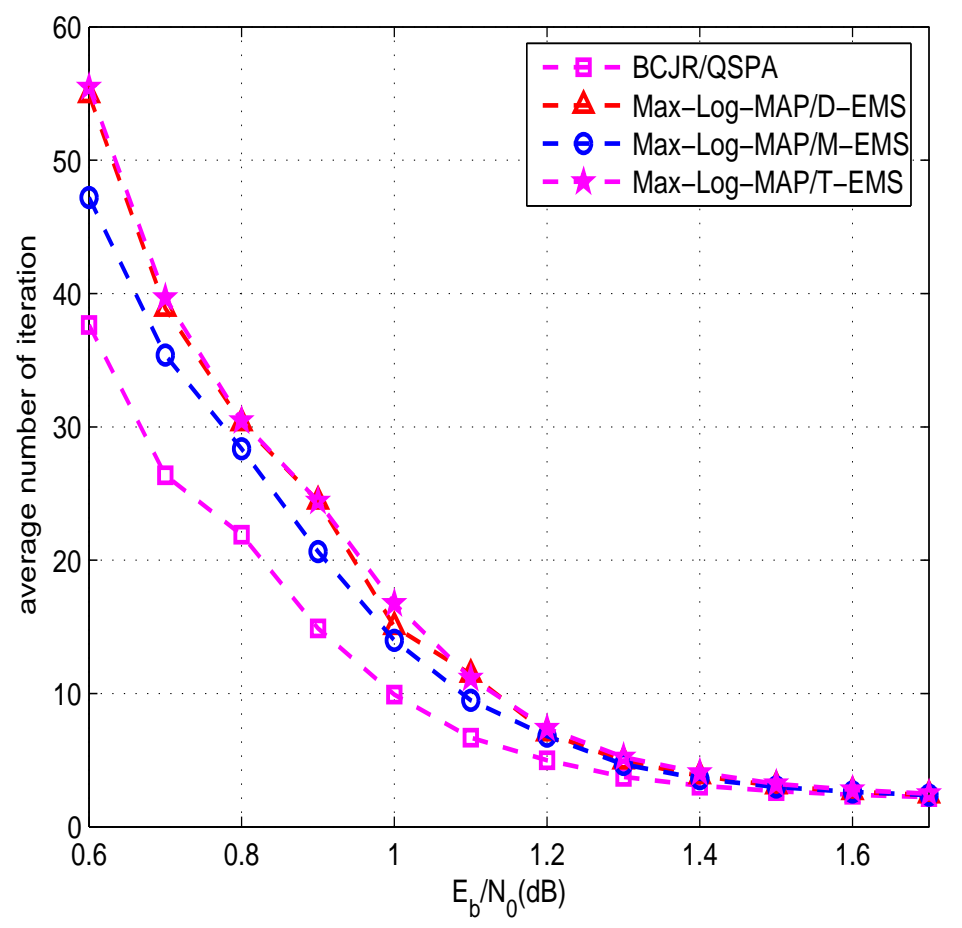

Figure 8: Average number of iteration for different algorithm in the $\mathcal{C}_{16}[225,173]$ coded H2RC system.

of different algorithms. It can be seen that, in low $E_{b} / N_{0}$ region, the average iteration numbers of Max-Log-Map/X-EMS are slightly larger than those of BCJR/QSPA, while in high $E_{b} / N_{0}$ region the average iteration numbers of Max-Log-Map/X-EMS are almost equal to those of BCJR/QSPA.

\section{Conclusions}

In this paper, we have proposed the Max-Log-MAP/X-EMS algorithm for NB-LDPC coded PRCPM system. Simulation results show that the MaxLog-MAP / $X$-EMS algorithm performs as well as the traditional BCJR/QSPA 
algorithm, but with reduced complexity. In addition, to show the advantages of the proposed serially concatenated system, we have compared the error performance of an NB-LDPC coded PRCPM system with that of an eBCH coded PRCPM system.

\section{References}

[1] J. B. Anderson, T. Aulin, C. E. Sundberg, Digital phase modulation, Springer, New York, USA, 1986, pp. 474-482.

[2] T. Aulin, C. E. Sundberg, Continuous phase modulation-part I: Full response signaling, IEEE Trans. Commun. 29 (1981) 196-209.

[3] T. Aulin, N. Rydbeck, C. E. Sundberg, Continuous phase modulationpart II: Partial response signaling, IEEE Trans. Commun. 29 (1981) 210-225.

[4] B. E. Rimoldi, A decomposition approach to CPM, IEEE Trans. Inform. Theory 34 (1988) 260-270.

[5] M. Xiao, T. Aulin, On analysis and design of low density generator matrix codes for continuous phase modulation, IEEE Trans. Wireless Commun. 6 (2007) 3440-3449.

[6] P. Moqvist, T. M. Aulin, Serially concatenated continuous phase modulation with iterative decoding, IEEE Trans. Commun. 49 (2001) 19011915. 
[7] M. Xiao, T. Aulin, Serially concatenated continuous phase modulation with convolutional codes over rings, IEEE Trans. Commun. 54 (2006) $1387-1396$.

[8] A. G. i Amat, C. A. Nour, C. Douillard, Serially concatenated continuous phase modulation with extended $\mathrm{BCH}$ codes, IEEE Information Theory Workshop on Information Theory for Wireless Networks (2007).

[9] A. G. I. Amat, C. A. Nour, C. Douillard, Serially concatenated continuous phase modulation for satellite communications, IEEE Trans. Wireless Commun. 8 (2009) 3260-3269.

[10] K. R. Narayanan, I. Altunbas, R. Narayanaswami, Design of serial concatenated msk schemes based on density evolution, IEEE Trans. Commun. 51 (2003) 1283-1295.

[11] T. Benaddi, C. Poulliat, M.-L. Boucheret, B. Gadat, G. Lesthievent, Protograph-based LDPC convolutional codes for continuous phase modulation, IEEE International Conference on Communications (ICC) (2015).

[12] X. Liu, C. Liang, X. Ma, Block Markov superposition transmission of convolutional codes with minimum shift keying signalling, IET Communications 9 (2015) 71-77.

[13] R. G. Gallager, Low-Density Parity-Check Codes, MIT Press, 1963.

[14] M. C. Davey, D. J. C. MacKay, Low-density parity check codes over GF $(q)$, IEEE Communications Letters 2 (1998) 165-167. 
[15] A. Voicila, D. Declercq, F. Verdier, et al, Low-complexity decoding for non-binary LDPC codes in high order fields, IEEE Trans. Commun. 58 (2010) 1365-1375.

[16] X. Ma, K. Zhang, H. Chen, Low complexity X-EMS algorithms for nonbinary LDPC codes, IEEE Trans. Commun. 60 (2012) 9-13.

[17] S. Zhao, Z. Lu, X. Ma, B. Bai, A variant of the EMS decoding algorithm for nonbinary LDPC codes, Communications Letters, IEEE 17 (2013) 1640-1643.

[18] K. Huang, D. Zhang, S. Zhao, X. Ma, Joint timing recovery and decoding algorithms for non-binary ldpc coded systems, Digital Signal Processing 23 (2013) 1115-1120.

[19] S. Zhao, Z. Lu, X. Ma, B. Bai, Joint detection/decoding algorithms for non-binary low-density parity-check codes over inter-symbol interference channels, IET Communications 7 (2013) 1522-1531.

[20] P. Robertson, E. Willebrun, P. Hoeher, A comparison of optimal and sub-optimal MAP decoding algorithms operating in the log domain, in: IEEE International Conference on Communications, Seattle, 1995, pp. 1009-1013.

[21] R. L. Bahl, F. J. J. Cocke, J. Raviv, Optimal decoding of linear codes for minimizing symbol error rate, IEEE Trans. Inform. Theory 20 (1974) $284-287$. 
[22] C. Berrou, A. Glavieux, P. Thitimajshima, Near shannon limit errorcorrecting coding and decoding: Turbo-codes, IEEE International Conference on Communications (1993) 1064-1070.

[23] J. Huber, W. Liu, An alternative approach to reduced-complexity CPMreceivers, IEEE J. Select. Areas Commun. 7 (1989) 1437-1449.

[24] S. Zhao, X. Ma, B. Bai, A class of nonbinary LDPC codes with fast encoding and decoding algorithms, IEEE Trans. Commun. 61 (2013) $1-6$.

[25] S. Lin, S. Song, L. Lan, constructions of nonbinary quasi-cyclic LDPC codes: a finite field approach, IEEE Trans. Commun. 56 (2008) 545-554.

Xiying Liu was born in Hunan province, China. She is currently pursuing her PhD degree at the Sun Yat-sen University. Her current research interests include digital phase modulation and channel coding.

Shancheng Zhao was born in Sichuan province, China. He is an assistant professor in Jinan university. His current research interests include design and decoding of non-binary LDPC codes and their applications.

Xiao Ma received the $\mathrm{PhD}$ degree in communication and information systems from Xidian University, China, in 2000. From 2000 to 2002, he was a Postdoctoral Fellow with Harvard University. From 2002 to 2004, he was a 
Research Fellow with City University of Hong Kong. Since 2004, he has been a Professor with the Department of Electronics and Communication Engineering, Sun Yat-sen University, Guangzhou, China. His research interests include information theory, channel coding theory and their applications to communication systems and digital recording systems. 
Xiying Liu was born in Hunan province, China. She is currently pursuing her PhD degree at the Sun Yat-sen University. Her current research interests include digital phase modulation and channel coding.

Shancheng Zhao was born in Sichuan province, China. He is an assistant professor in Jinan university. His current research interests include design and decoding of non-binary LDPC codes and their applications.

Xiao Ma received the $\mathrm{PhD}$ degree in communication and information systems from Xidian University, China, in 2000. From 2000 to 2002, he was a Postdoctoral Fellow with Harvard University. From 2002 to 2004, he was a Research Fellow with City University of Hong Kong. Since 2004, he has been a Professor with the Department of Electronics and Communication Engineering, Sun Yat-sen University, Guangzhou, China. His research interests include information theory, channel coding theory and their applications to communication systems and digital recording systems. 


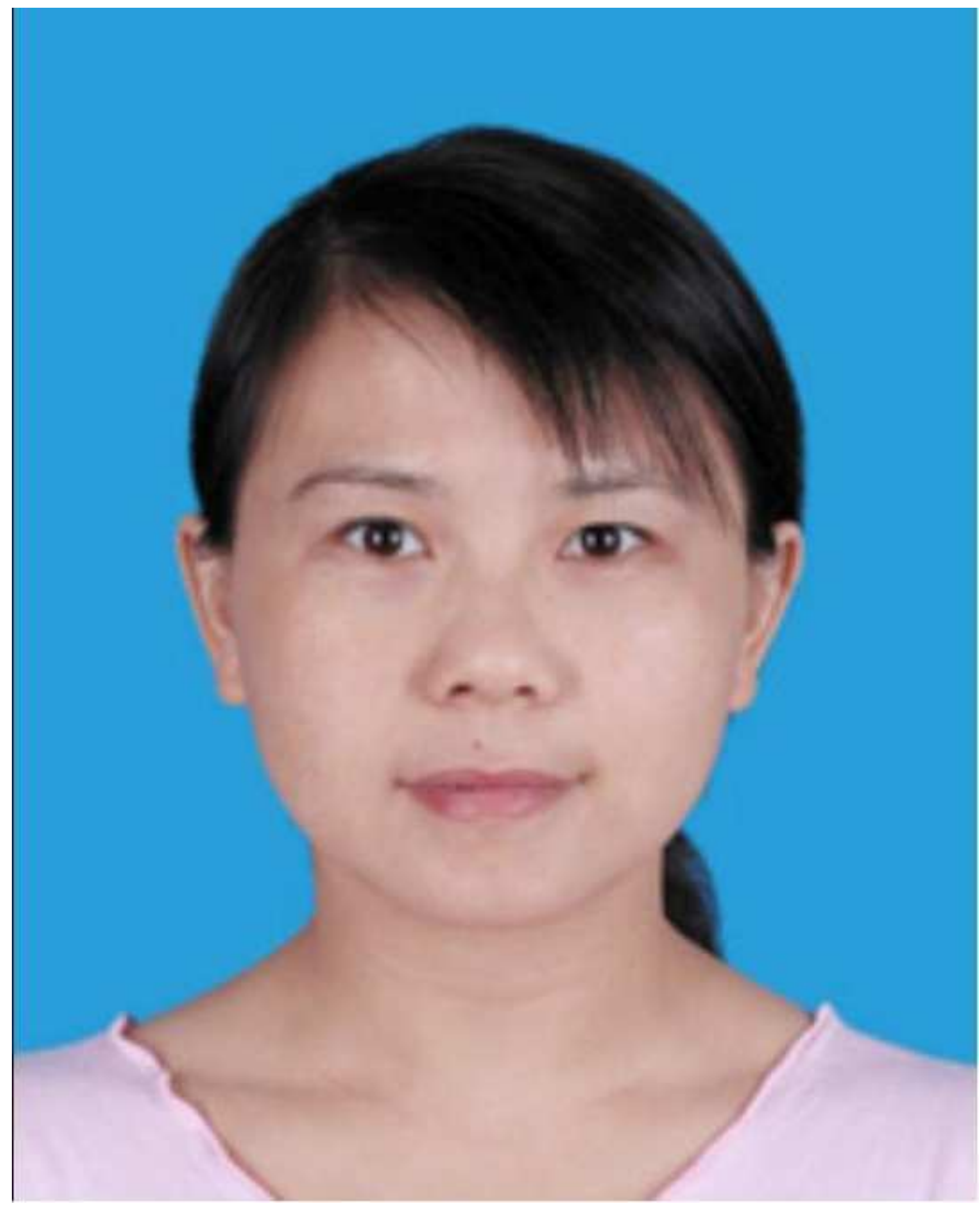


